\title{
On second order consensus protocols allowing joint-agent interactions
}

\author{
A. Tesi $^{1}$ and D. Angeli ${ }^{2}$
}

\begin{abstract}
In this note, we propose a second order consensus protocol allowing joint-agent interactions. Our analysis is addressed to networks where agents are grouped in interaction triplets. A triplet is a set of three distinct agents, where the interaction dynamics is such that one member is sensitive to the influence brought by the other two only when it receives simultaneous and consistent opinions from them. This allows the network to reach a second order consensus configuration more robustly, even in the presence of exogenous disturbances on the dynamics of one or more agents. The piecewise linear function used to model the robust interaction within a triplet leads to the energy of the interaction being conserved along certain directions, as if some agents were connected by virtual springs. In conclusion, a convergence analysis is performed by means of the recursive detection of such conservation laws.
\end{abstract}

\section{INTRODUCTION AND MOTIVATIONS}

Consensus protocols have been extensively studied by the scientific community in recent years. These are distributed algorithms that allow a network of interacting agents to achieve an agreement, also called consensus equilibrium, upon a certain quantity of interest. The increasing emphasis on similar protocols is motivated by their vast field of application, which encompasses sensor and communication networks [1][3], coordination problems [4], cooperative control [5]-[6] and formation control [7]-[9].

Interesting papers have been devoted to investigate necessary and sufficient topological conditions for the information being spread across the network in such a way that a consensus configuration emerges. In [10], the analysis deals with specific scenarios, such as directed networks with fixed or switching topologies and time delays, whereas in [11] a graph theoretical condition is formulated concerning the strength of the interaction. In both works, the proposed protocol is in the form of a linear time-dependent equation. Similar results have been generalized to nonlinear scenarios [12]-[13].

Furthermore, a second order extension of linear consensus protocols is presented by Wei Ren in [14]. These algorithms enable a population of second order systems to reach an agreement upon a variable of interest and its derivative, within the considered set-up. We point out that second order consensus theory leads to remarkable benefits in formation

\footnotetext{
${ }^{1}$ A. Tesi is with the Chair of Cyber-Physical Systems in Production Engineering, Mechanical Engineering Department, Technical University of Munich, Germany. Email: alessandro.tesi@tum.de

${ }^{2}$ D. Angeli is with the Control and Power Group, Electric and Electronic Engineering Department, Imperial College London, UK and with the Department of Information Engineering, University of Florence, Italy. Email: d. angeli@imperial.ac.uk

We want to thank Prof. M. Caccamo and the Chair of Cyber-Physical Systems in Production Engineering, since it partially sponsored this work. Additionally, Alessandro Tesi is supported by Alexander von Humboldt funds, endowed by the German Federal Ministry of Education and Research.
}

control tasks. The individual behaviour of any vehicle, indeed, often complies with a second order model. In [15] some variants are proposed to deal with specific case studies, such as the absence of relative velocity measures, bounded control input, or group velocity tracking task. An extension to nonlinear scenarios is reported in [16].

However, an inherent issue of consensus protocols is the lack of robustness. When one agent shows an unexpected dynamics, either due to disturbances or deceiving purposes, the capability of the whole network to converge towards consensus is lost. After their study on consensus in nonlinear monotone networks with unilateral interactions [17], Angeli and Manfredi have introduced the concept of joint-agent interaction in a first order consensus problem [18]-[19]. By virtue of this form of interaction, every agent is not sensitive to the influence of any of its neighbours singularly taken, but updates its internal state variable only in response to simultaneous and coherent actions from them. As a result, every agent operates a cross validation of the information received, so as to accept and propagate only the trustworthy one. Therefore, consensus can be achieved more robustly, even when some agents are subjected to external disturbances or show a malicious dynamics. Borrowing the language of Petri Nets, they found in the non-existence of a pair of disjoint siphons a necessary and sufficient condition for a network to reach consensus for any choice of initial conditions. Our main contribution in this paper is the development of a second order consensus protocol allowing joint-agent interactions. A complete extension of [18] is not trivial; the concept of siphon, indeed, is no longer sufficient to fully characterize the opinion dynamics in a second order scenario, where the evolution of the variables of interest is in general not monotonic, because of the initial conditions of their derivatives. Therefore, the class of topologies to which our study is addressed has been restricted and a specific joint-agent interaction function, also suggested in [18], has been assumed. While leading to less general results from a theoretical standpoint, these assumptions have led to some novel tools for the convergence analysis and, by extension, to a preliminary validation of our second order consensus protocol allowing joint-agent interactions.

\section{PROBLEM FORMULATION}

The second order dynamics of a network of cooperating agents is represented by the following nonlinear finite dimensional system:

$$
\dot{\mathbf{x}}=\left[\begin{array}{c}
\dot{\mathbf{x}}^{p} \\
\dot{\mathbf{x}}^{v}
\end{array}\right]=\left[\begin{array}{c}
\mathbf{x}^{v} \\
\psi\left(\mathbf{x}^{p}\right)+\delta\left(\mathbf{x}^{v}\right)
\end{array}\right]
$$


where $e$ is the number of agents, $\mathbf{x}^{p}, \mathbf{x}^{v} \in \mathbb{R}^{e}$ denote the position and velocity variables, $\psi, \delta: \mathbb{R} \times \mathbb{R}^{e} \rightarrow \mathbb{R}^{e}$ encode the joint-agent interactions and a damping action respectively. The unique solution of (1) corresponding to initial condition $\mathbf{x}_{0}$ is denoted as $\phi\left(t, \mathbf{x}_{0}\right)$. Network (1) converges asymptotically to a second order consensus equilibrium when the following holds:

$$
\lim _{t \rightarrow \infty} \phi\left(t, \mathbf{x}_{0}\right)-\left[\begin{array}{c}
(\bar{p}+\bar{v} t) \mathbf{1} \\
\bar{v} \mathbf{1}
\end{array}\right]=0
$$

for some $\bar{p}, \bar{v} \in \mathbb{R}$, where $\mathbf{1}$ is the vector of all ones in $\mathbb{R}^{e}$. Furthermore, we say that a global asymptotic consensus is achieved when this occurs for any choice of initial conditions.

In [18], Angeli and Manfredi do not make any a priori restrictions concerning the topology of interactions, and rely on the language of Petri Nets and the concept of siphon to characterize occurrence of global asymptotic consensus. In a first order scenario, indeed, a crucial property holds for the system's evolution: the states of those agents having the maximum and the minimum initial beliefs do not monotonically increase and decrease, respectively. Similar considerations are crucial in their proof of convergence, but cease to be valid in our second order consensus problem, due to the role of initial velocities. Therefore, a requirement of symmetry on both the topology and the system's dynamics is adopted, so as to compensate the complexity and the potential instability produced by the lack of monotonicity.

Let $\mathcal{N}=\{1, \ldots, e\}$ be the set of agents and the operator $|\cdot|$ denote the cardinality of a set.

Definition 1. An interaction triplet is a set of agents $T \subset \mathcal{N}$ such that $|T|=3$.

The present study is addressed to topologies where agents are grouped by design in a set of $n$ interaction triplets, with $3 \leq e \leq 3 n: \mathcal{T}=\left\{T_{1}, \ldots, T_{n}\right\}=$ $\left\{\left\{r_{1}, s_{1}, t_{1}\right\}, \ldots,\left\{r_{n}, s_{n}, t_{n}\right\}\right\}$. Every agent directly interacts only with those which belong to its same triplet. Moreover, an agent is allowed to be a member of more than one triplet: in this case each membership will provide a potential contribution to its state updating. Our purpose is to set an interaction dynamics in such a way that a second order consensus configuration may emerge even in the presence of malicious agents or exogenous disturbances. In this regard, the choice of a triplet-based topology is in accordance with a feature of robustness: since a player needs to cross validate at least two inputs for its state updating, the minimal topological structure allowing joint-agent interactions is a set composed by three distinct agents. An agent $r_{i} \in T_{i}$ will be sensitive to the influence of $s_{i}, t_{i} \in T_{i}$ only when it receives a consistent and coherent action from them. From a mathematical standpoint, this form of interaction is modelled by the following function: $\mu_{\left\{s_{i}, t_{i}\right\} \rightarrow r_{i}}(\mathbf{x})$, or equivalently $\mu\left(x_{s_{i}}^{p}, x_{t_{i}}^{p}, x_{r_{i}}^{p}\right)$. Then, a balancing criterion is adopted in the assignment of the interaction dynamics. If an agent belongs to only one triplet, $r_{i} \in T_{i}$, its second order dynamics is defined as:

$$
\ddot{x}_{r_{i}}^{p}=\dot{x}_{r_{i}}^{v}=\lambda \mu\left(x_{s_{i}}^{p}, x_{t_{i}}^{p}, x_{r_{i}}^{p}\right)-\epsilon x_{r_{i}}^{v},
$$

whereas if an agent belongs to $m$ triplets, $r \in T_{1}, \ldots, T_{m}$, its internal belief is updated according to:

$$
\ddot{x}_{r}^{p}=\dot{x}_{r}^{v}=\frac{\lambda}{m} \sum_{k=1}^{m} \mu\left(x_{s_{k}}^{p}, x_{t_{k}}^{p}, x_{r}^{p}\right)-\epsilon x_{r}^{v} .
$$

Notice that $\lambda>0$, and $\epsilon>0$ is a damping coefficient. The knowledge of the topology and rules (2) and (3) define the explicit structures of $\psi$ and $\delta$.

At this point, we borrow the shape of $\mu$ from Angeli and Manfredi [18]:

$$
\begin{gathered}
\mu\left(x_{s_{i}}^{p}, x_{t_{i}}^{p}, x_{r_{i}}^{p}\right):=\max \left\{\min \left(x_{s_{i}}^{p}-x_{r_{i}}^{p}, 0\right), \min \left(x_{t_{i}}^{p}-x_{r_{i}}^{p}, 0\right)\right\} \\
+\min \left\{\max \left(x_{s_{i}}^{p}-x_{r_{i}}^{p}, 0\right), \max \left(x_{t_{i}}^{p}-x_{r_{i}}^{p}, 0\right)\right\} .
\end{gathered}
$$

This piecewise linear function is positive when $x_{s_{i}}^{p}, x_{t_{i}}^{p}>x_{r_{i}}^{p}$; it is negative when $x_{s_{i}}^{p}, x_{t_{i}}^{p}<x_{r_{i}}^{p}$. As a consequence, in both scenarios the action of $\mu$ attracts the position state of $r_{i}$ towards the interval defined by $x_{s_{i}}^{p}$ and $x_{t_{i}}^{p}$. On the other hand, when $x_{r_{i}}^{p}$ lies in that interval, the function is equal to zero and the influence is deactivated.

Notice that from this point forward, the following conventions will be adopted, with a slight abuse, in order to simplify the notation:

$$
x_{i}=x_{i}^{p}, \quad \dot{x}_{i}={\dot{x_{i}}}^{p}=x_{i}^{v}, \quad \ddot{x}_{i}=\ddot{x}_{i}^{p}=\dot{x}_{i}^{v} .
$$

Moreover, $\phi\left(t, \mathbf{x}_{0}, \dot{\mathbf{x}}_{0}\right)$ will denote the unique solution for $\mathbf{x}$ of equation

$$
\ddot{\mathbf{x}}=\psi(\mathbf{x})+\delta(\dot{\mathbf{x}})
$$

corresponding to initial conditions $\mathbf{x}_{0}$ and $\dot{\mathbf{x}}_{0}$.

In the conclusion of this Section, a remark is needed. Our convergence analysis is based on the recursive detection of conservation laws within the interaction dynamics. On this regard, the restriction to triplet-based topologies and the assignment of a balanced dynamics are sufficient conditions for such conservation laws to emerge. Nevertheless, similar assumptions might not be necessary.

\section{CONSERVATIVE INTERACTIONS}

The choice of a piecewise linear function to model the robust interaction among three agents leads $\psi$ to admit linear eigenfunctions of the Koopman operator. This is due to a property shown by $\mu$ in the context of a triplet, as reported by the following lemma. Proof is omitted for brevity.

Lemma 1. Provided a triplet $T_{i}=\left\{r_{i}, s_{i}, t_{i}\right\}$, it holds:

$$
\mu\left(x_{r_{i}}, x_{s_{i}}, x_{t_{i}}\right)-\mu\left(x_{r_{i}}, x_{t_{i}}, x_{s_{i}}\right)=-\left(x_{t_{i}}-x_{s_{i}}\right) .
$$

\section{A. Virtual springs}

At this point, we introduce the crucial tool for our convergence analysis, resuming conventions (4).

Definition 2. A virtual spring is a vector $\mathbf{s}^{\top} \in \mathbb{R}^{e}$ such that:

$$
\mathbf{s}^{\top} \psi(\mathbf{x})=-\lambda \mathbf{s}^{\top} \mathbf{x}, \quad \lambda>0 \text {. }
$$


When such $\mathbf{s}^{\top}$ exists, the energy of the interaction along that specific direction is conserved. However, in the system's dynamics the interaction force is combined with a damping term and this energy is gradually dissipated. As a result, we obtain the typical equation of a damped oscillator:

$$
\mathbf{s}^{\top}(\ddot{\mathbf{x}}+\epsilon \dot{\mathbf{x}}+\lambda \mathbf{x})=0 .
$$

We provide an example for the sake of clarity. Let us consider the network $\mathcal{T}=\{\{1,2,3\},\{1,2,4\}\}$. Its second order dynamics is expressed by the following set of differential equations:

$$
\left\{\begin{array}{l}
\ddot{x}_{1}=\frac{\lambda \mu\left(x_{2}, x_{3}, x_{1}\right)}{2}+\frac{\lambda \mu\left(x_{2}, x_{4}, x_{1}\right)}{2}-\epsilon \dot{x}_{1} \\
\ddot{x}_{2}=\frac{\lambda \mu\left(x_{1}, x_{3}, x_{2}\right)}{2}+\frac{\lambda \mu\left(x_{1}, x_{4}, x_{2}\right)}{2}-\epsilon \dot{x}_{2} \\
\ddot{x}_{3}=\lambda \mu\left(x_{1}, x_{2}, x_{3}\right)-\epsilon \dot{x}_{3} \\
\ddot{x}_{4}=\lambda \mu\left(x_{1}, x_{2}, x_{4}\right)-\epsilon \dot{x}_{4} .
\end{array}\right.
$$

Lemma 1 ensures that $\mathbf{s}_{1}^{\top}=[1,-1,0,0]$ is a virtual spring. Similarly, $\mathbf{s}_{2}^{\top}=[2,0,-1,-1]$ is a virtual spring. Notice that the linear combination of springs is again a spring, like $\mathbf{s}_{3}^{\top}=[0,2,-1,-1]$.

\section{B. Rule for spring detection}

Since virtual springs play a key role in the proof of convergence towards a consensus equilibrium, an interesting point is the synthesis of a rule which allows for such springs being detected in a general topology. Notice that some disjoint rules based on the form of the set of triplets can be easily provided for verifying the presence of springs by inspection. However, in this work a more general unified rule, suitable for an algorithmic implementation, will be presented.

Let us map network $\mathcal{T}$ in a graph $G=(N, E, m)$. Every agent is associated with a node and $N=\left\{n_{1}, \ldots, n_{e}\right\}$ is the set of nodes. Since a one-to-one correspondence exists between agents and nodes, $n_{i} \in N$ will be referred as node and agent equivalently. The multiplicity of a node $n_{i} \in N$ is denoted as $q\left(n_{i}\right)$ and is defined as the number of triplets which it belongs to. By extension, the joint-multiplicity of $n_{i}, n_{j} \in N$ is denoted as $q\left(n_{i}, n_{j}\right)$ and is defined as the number of triplets which they appear in simultaneously. The edge set is $E=\left\{e_{i j}=\left(n_{i}, n_{j}\right) \mid q\left(n_{i}, n_{j}\right) \neq 0\right\}$, while $m_{i}$ is a weight on node $n_{i}$ equal to its multiplicity.

Let us define $G^{*}=\left(N^{*}, E^{*}, m^{*}\right)$ such that $N^{*}=$ $\left\{n_{1}^{*}, \ldots, n_{c}^{*}\right\} \subset N, E^{*}=\left\{e_{i j}^{*}=\left(n_{i}^{*}, n_{j}^{*}\right) \mid q\left(n_{i}^{*}, n_{j}^{*}\right) \neq 0\right\} \subset$ $E$ and $m_{i}^{*}$ is a signed weight on node $n_{i}^{*}$, with $\left|m_{i}^{*}\right|=m_{i}$.

Rule 1. A graph $G^{*}=\left(N^{*}, E^{*}, m^{*}\right)$ identifies a virtual spring in network $\mathcal{T}$ if:

1) $\forall n_{i}^{*}, n_{j}^{*} \in N^{*}$ the following implication holds

$$
q\left(n_{i}^{*}, n_{j}^{*}\right) \neq 0 \Rightarrow \frac{m_{i}^{*}}{\left|m_{i}^{*}\right|}=-\frac{m_{j}^{*}}{\left|m_{j}^{*}\right|}
$$

2) $\forall n_{i}^{*} \in N^{*}$ it holds

$$
\sum_{j \neq i} q\left(n_{i}^{*}, n_{j}^{*}\right)=\left|m_{i}^{*}\right| .
$$

The virtual spring is a vector $\mathbf{s}^{\top} \in \mathbb{R}^{e}$ of support equal to $N^{*}$ and of value $m_{i}^{*}$ in each $n_{i}^{*}$-th entry (and 0 in all the others).

In other words, (6) says that if two agents in $N^{*}$ belong together to one or more triplets, they must be assigned discordant weights. Condition (7) instead, refers to a balancing between the number of interactions offered by $n_{i}^{*}$ and the number of those brought by the other nodes.

Proof. Let us build $s^{\top}$ as in the rule statement. We denote with $\psi_{n_{i}^{*}}(\mathbf{x})$ the $n_{i}^{*}$-th component of $\psi$. Furthermore, let us consider $n_{i}^{*} \in N^{*}$ and define the set $H^{i}:=\left\{n_{j}^{*} \in N^{*}, j \neq\right.$ $\left.i \mid q\left(n_{i}^{*}, n_{j}^{*}\right) \neq 0\right\}=\left\{h_{1}^{i}, \ldots, h_{k_{i}}^{i}\right\}$. Condition (7) ensures that $H^{i} \neq \emptyset$. Agents $n_{i}^{*}$ and $h_{l}^{i}$, with $1 \leq l \leq k_{i}$, are shared by $q\left(n_{i}^{*}, h_{l}^{i}\right)$ triplets; in each one of them a third agent appears, but condition (6) ensures that it does not belong to $N^{*}$. Triplet by triplet, the identity of the third agent is not relevant in the synthesis of a spring; therefore, we write:

$$
\psi_{n_{i}^{*}}(\mathbf{x})=\frac{\lambda}{\left|m_{i}^{*}\right|} \sum_{l=1}^{k_{i}} q\left(n_{i}^{*}, h_{l}^{i}\right) \mu\left(\cdot, x_{h_{l}^{i}}, x_{n_{i}^{*}}\right)
$$

where "." is used to replace the state of the third agent in every addend. The generic $\mu\left(\cdot, x_{h_{l}^{i}}, x_{n_{i}^{*}}\right)$ is available for pairing with a proper counterpart so as to produce a linear elastic term according to (5). At this point, we may write:

$$
\mathbf{s}^{\top} \psi(\mathbf{x})=\lambda \sum_{n_{i}^{*} \in N^{*}} \sum_{l=1}^{k_{i}} \frac{m_{i}^{*}}{\left|m_{i}^{*}\right|} q\left(n_{i}^{*}, h_{l}^{i}\right) \mu\left(\cdot, x_{h_{l}^{i}}, x_{n_{i}^{*}}\right) .
$$

The right-hand side contains all the paired differences requested by Lemma 1 and needed to prove our thesis. In fact, the consistency of signs is ensured by (6). Moreover, the symmetry is guaranteed by the implication: $n_{j}^{*} \in H^{i} \Rightarrow$ $n_{i}^{*} \in H^{j}$. As a result, (8) becomes:

$$
\mathbf{s}^{\top} \psi(\mathbf{x})=-\lambda \sum_{n_{i}^{*} \in N^{*}} m_{i}^{*} x_{n_{i}^{*}}=-\lambda \mathbf{s}^{\top} \mathbf{x} .
$$

\section{Algorithm for spring detection}

The goal of this Subsection is to formulate an algorithm to perform automatically the spring search following Rule 1. Let $\mathcal{T}$ be a triplet-based network and $G$ its graph. We pick a node $n_{i} \in N$ and we look for all the springs in which it appears. We recall that finding a spring means determining its support and nonzero entries or, equivalently, identifying the related graph. From (5), node $n_{i}$ has a binding capacity, given by the number and the identities of the $\mu$-contributions appearing in its interaction dynamics. As suggested by (7), in the synthesis of a spring this binding capacity needs to be saturated by the addition of one or more nodes from $N$. Moreover, this saturation process must prevent the completion of a triplet, according to (6). If, after their addition, the new nodes still have a residual binding capacity, they will undergo the same saturation procedure simultaneously. We point out that the set of additional nodes which guarantees the saturation may not be unique. For this reason, in order to explore 
all the possible options, a graph of graphs is progressively developed: $M^{i}=\left(F^{i}, A^{i}\right)$, with $F_{j}^{i}=\left\{F_{j}^{i}, j=1, \ldots\right\}$ and $A^{i}=\left\{a_{j k}^{i}=\left(F_{j}^{i}, F_{k}^{i}\right) \mid F_{j}^{i}, F_{k}^{i} \in F^{i}\right\}$. The generic $F_{j}^{i}$ is a graph $\left(K_{j}, L_{j}, m, c\right)$, where $K_{j} \subset N$ and $L_{j}$ are the node and the edge sets respectively, $m$ is a signed weight on nodes and $c$ is the residual capacity of nodes.

In the first step $M^{i}$ is initialized: $M^{i}=\left(F_{0}^{i}, \emptyset\right)$, with $K_{0}:=\left\{n_{i}\right\}, L_{0}:=\emptyset, m\left(n_{i}\right):=q\left(n_{i}\right)$ and $c\left(n_{i}\right):=m\left(n_{i}\right)$.

We say that a node in $K_{j}$ is unbalanced when its residual capacity is not equal to zero, i.e. it has not been saturated yet. A graph $F_{j}^{i}$ is said unbalanced when at least one of its nodes is. Our strategy consists in expanding $M^{i}$ so as to perform a progressive balancing of its nodes. For instance, let us consider the balancing procedure for $F_{j}^{i}$. The set of its unbalanced nodes is $U_{j}=\left\{u_{p} \in K_{j} \mid c\left(u_{p}\right)>0\right\}=$ $\left\{u_{1}, \ldots, u_{q}\right\}$. A balancing group of $u_{p} \in U_{j}$ is a set $B\left(u_{p}\right)=$ $\left\{b_{1}, \ldots, b_{r}\right\} \subset N$ such that:

1) $\forall b_{s} \in B\left(u_{p}\right)$, it holds

$$
q\left(u_{p}, b_{s}\right) \neq 0
$$

2) the following condition is met

$$
\sum_{s=1}^{r} q\left(u_{p}, b_{r}\right)=c\left(u_{p}\right)
$$

3) $1 \leq k \leq n$, it holds

$$
T_{k} \not \subset K_{j} \cup B\left(u_{p}\right) \text {. }
$$

Condition (9) requires every element of $B\left(u_{p}\right)$ to interact with $u_{p}$, (10) guarantees that the whole group is able to saturate the residual capacity of $u_{p}$, while (11) avoids triplet completions. In general, there may be more options for the balancing of a single node, so $u_{p}$ admits a family of balancing groups: $\mathcal{B}\left(u_{p}\right)=\left\{B_{1}\left(u_{p}\right), \ldots, B_{s_{p}}\left(u_{p}\right)\right\}$. The balancing of $F_{j}^{i}$ requires all of its $q$ unbalanced nodes to be simultaneously balanced. Therefore, $\pi=\prod_{t=1}^{q} s_{t}$ balancing combinations exist for $F_{j}^{i}$. However, only $\pi^{*} \leq \pi$ combinations are acceptable: those whose addition to the current node set would involve the completion of a triplet must be discarded due to (6). Let us denote the w-th acceptable combination with $C_{w}=\left\{B\left(u_{1}\right), \ldots, B\left(u_{q}\right)\right\}$. Algorithm 1 summarizes the balancing of $F_{j}^{i}$ and the consequent development of $M^{i}$.

A graph $F_{j}^{i}$ will not have any children only when it does not admit any balancing group or when it is already balanced. When the second is the case, a spring has been detected: its support is defined by the node set $K_{j}$ while the related nonzero entries are given by the signed weights. The whole set of virtual springs in $\mathcal{T}$ can be found electing as a root one node of $N$ at a time.

\section{Convergence Analysis}

As a result of damping, a virtual spring asymptotically enforces an algebraic constraint over the system's dynamics. If a set of $m$ independent springs is detected in a network $\mathcal{T}$, under the assumption of bounded solutions $\phi\left(t, \mathbf{x}_{0}, \dot{\mathbf{x}}_{0}\right)$ it

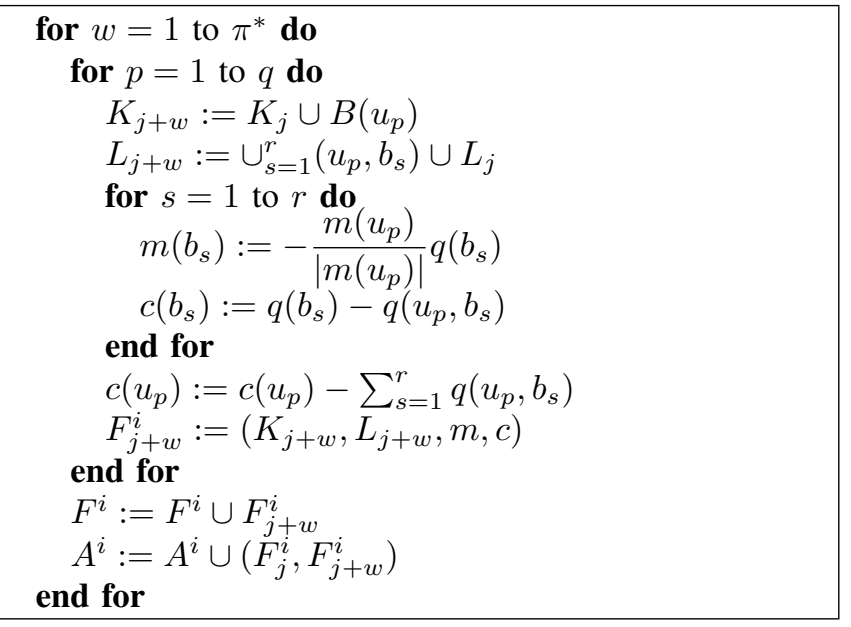

Fig. 1: Algorithm for balancing of $F_{j}^{i}$

holds:

$$
\lim _{t \rightarrow \infty}\left[\begin{array}{c}
\mathbf{s}_{1}^{\top} \\
\vdots \\
s_{m}^{\top}
\end{array}\right] \phi\left(t, \mathbf{x}_{0}, \dot{\mathbf{x}}_{0}\right):=\lim _{t \rightarrow \infty} S \phi\left(t, \mathbf{x}_{0}, \dot{\mathbf{x}}_{0}\right)=0 .
$$

In the light of this, only $r:=e-m$ among the $\mathbf{x}$ variables will be algebraically independent. As clarified in the following, this set of independent variables can be isolated via a proper change of coordinates. Their second order dynamics can be studied and will be called residual dynamics. At this level, we only remark that the residual dynamics evolves in the subspace $\operatorname{ker}(S)$. Notice that $\mathbf{1} \in \operatorname{ker}(S)$, being a right eigenvector associated to the null eigenvalue. The latter fact is inferred from Rule 1 and is an expression of the translation invariance property of the system. When $\operatorname{ker}(S)=\operatorname{span}(\mathbf{1})$, the system is constrained on a second order consensus trajectory. On the other hand, when $m<e-1$ the convergence towards consensus is not guaranteed, due to $\operatorname{dim}[\operatorname{ker}(S)]>1$. In this scenario, the analysis of convergence is performed by searching further conservation laws in the subspace of the residual dynamics. Again, as a result of damping, after the achievement of steady state conditions they will provide additional constrains and the dimension of the residual dynamics will be further reduced.

\section{A. Virtual springs in the residual dynamics}

We define a matrix $K \in \mathbb{R}^{e \times r}$, with $r=e-m$, whose columns are a basis of $\operatorname{ker}(S)$ :

$$
K=\left[\begin{array}{llll}
\mathbf{k}_{1} & \ldots & \mathbf{k}_{r-1} & \mathbf{1}
\end{array}\right] .
$$

Let us operate the following change of coordinates:

$$
\mathbf{z}=\left[\begin{array}{l}
\mathbf{y} \\
\alpha
\end{array}\right]=\left[\begin{array}{c}
S \\
\left(K^{\top} K\right)^{-1} K^{\top}
\end{array}\right] \mathbf{x} .
$$

Such a space decomposition results in the residual dynamics being described by the only $\alpha$ coordinates:

$$
\ddot{\alpha}=\left(K^{\top} K\right)^{-1} K^{\top}[\psi(K \alpha)+\delta(K \dot{\alpha})] .
$$


Definition 3. A virtual spring in the subspace of the residual dynamics is a vector $\mathbf{c}^{\top}$ such that:

$$
\mathbf{c}^{\top}\left(K^{\top} K\right)^{-1} K^{\top} \psi(K \alpha)=-\lambda \mathbf{c}^{\top} \alpha, \lambda>0 .
$$

When such a vector exists, it indicates a direction in $\operatorname{ker}(S)$ along which the energy of the interaction is conserved. A damped oscillation occurs as a result of dissipation:

$$
\mathbf{c}^{\top}[\ddot{\alpha}+\epsilon \dot{\alpha}+\lambda \alpha]=0 .
$$

Next we formulate a necessary and sufficient condition for a vector to be a virtual spring in the residual dynamics. To this purpose, we define the set of all possible inequality relations among $\mathbf{x}$ coordinates as $O(\mathbf{x})=\left\{o_{1}(\mathbf{x}), \ldots, o_{e} !(\mathbf{x})\right\}$. The set $R=\left\{R_{i} \mid R_{i}=\left\{\mathbf{x} \in \mathbb{R}^{e} \mid o_{i}(\mathbf{x})\right\}, i=1, \ldots e !\right\}$ is the partition of $\mathbb{R}^{e}$ induced by $O(\mathbf{x})$. Then, we adopt a partition of $\operatorname{ker}(S): P=\left\{P_{i} \mid P_{i}=R_{j} \cap \operatorname{ker}(S) \neq \emptyset, R_{j} \in R\right\}$, $|P|=a$. The Jacobian of $\psi$ with respect to $\alpha$, computed in $P_{i}$ and expressed in $\alpha$ coordinates is

$$
J_{P_{i}}:=\left.\left(K^{\top} K\right)^{-1} K^{\top} \frac{\partial \psi(K \alpha)}{\partial \alpha} K\right|_{K \alpha \in P_{i}} .
$$

Lemma 2. A vector $\mathrm{c}^{\top}$ is a virtual spring in the subspace of the residual dynamics if and only if:

$$
\mathbf{c}^{\top}\left[\begin{array}{lll}
J_{P_{1}}+\lambda I & \ldots & J_{P_{a}}+\lambda I
\end{array}\right]=0 .
$$

Proof. By computing the derivative of (14) with respect to $\alpha$ in one region $P_{i} \in P$, we obtain: $\mathbf{c}^{\top}\left[J_{P_{i}}+\lambda I\right]=0$. Since $P$ is a partition of $\operatorname{ker}(S)$, our thesis follows.

The leading principal minor of maximum order of $J_{P_{i}}$ is denoted as $\tilde{J}_{P_{i}}$. Our system is translation invariant; moreover, our choice of $K$ brings to $\ddot{\alpha}$ being independent from $\alpha_{r}$. In the light of this,

$$
\mathbf{c}^{\top}\left[\begin{array}{lll}
\tilde{J}_{P_{1}}+\lambda I & \ldots & \tilde{J}_{P_{a}}+\lambda I
\end{array}\right]=0
$$

will be used instead of (15) as a systematic tool for searching virtual springs and damped oscillations in the subspace of residual dynamics.

When $\mathbf{c}^{\top}$ is detected, it is transformed in the original set of coordinates according to (13), and the notation $\mathbf{s}^{\top}$ is resumed; an additional constraint over the system's dynamics is enforced after the transient phase has expired.

\section{B. A cascade approach}

The analysis technique of the residual dynamics allows for the convergence study to be carried out by means of a cascade approach. Let us consider a generic network $\mathcal{T}$. In the first stage, $m_{1}$ independent springs are detected by Rule 1: $\mathbf{s}_{1,1}^{\top}, \ldots, \mathbf{s}_{1, m_{1}}^{\top}$. We set $S_{1}=\left[\mathbf{s}_{1,1} \ldots \mathbf{s}_{1, m_{1}}\right]^{\top}$ and we initialize $S:=S_{1}$. If $\operatorname{ker}(S)=\operatorname{span}(\mathbf{1})$, the procedure is arrested and the global convergence towards consensus is proved; otherwise, a second stage is needed. In the latter scenario, a basis of $\operatorname{ker}(S)$ is stored in $K$ according to (12) and the change of coordinates (13) is adopted. If no springs emerge from the analysis of the residual dynamics, the procedure is arrested and the global convergence towards consensus is not proved. Conversely, if a set of $m_{2}$ independent springs is found, we set $S_{2}=$ $\left[\mathbf{s}_{2,1} \ldots \mathbf{s}_{2, m_{2}}\right]^{\top}$ and we update:

$$
S:=\left[\begin{array}{l}
S_{1} \\
S_{2}
\end{array}\right] \text {. }
$$

A new test on $\operatorname{ker}(S)$ is performed, and the procedure is repeated in an iterative fashion. The procedure is arrested at stage $n$, with $S_{n}=\left[\mathbf{s}_{n, 1} \ldots \mathbf{s}_{n, m_{n}}\right]^{\top}$ and

$$
S:=\left[\begin{array}{c}
S_{1} \\
\vdots \\
S_{n}
\end{array}\right],
$$

either because $\operatorname{ker}(S)=\operatorname{span}(\mathbf{1})$ or $\operatorname{ker}(S) \neq \operatorname{span}(\mathbf{1})$ and no further springs emerge from the analysis of the residual dynamics. In the first case the convergence is proved, in the second it is not. Notice that a similar approach corresponds to the following decomposition of the system's dynamics:

$$
\left\{\begin{aligned}
S_{1} \ddot{\mathbf{x}}+\epsilon S_{1} \dot{\mathbf{x}}+\Lambda_{1} S_{1} \mathbf{x} & =0 \\
S_{2} \ddot{\mathbf{x}}+\epsilon S_{2} \dot{\mathbf{x}}+\Lambda_{2} S_{2} \mathbf{x} & =\tilde{g}_{1}(\mathbf{x}) \\
& \vdots \\
S_{n} \ddot{\mathbf{x}}+\epsilon S_{n} \dot{\mathbf{x}}+\Lambda_{n} S_{n} \mathbf{x} & =\tilde{g}_{n-1}(\mathbf{x})
\end{aligned}\right.
$$

where $\Lambda_{i} \in \mathbb{R}^{m_{i} \times m_{i}}$ and $\tilde{g}_{i}$ is an external force produced by the transient phases of all the previous stages, such that $\tilde{g}_{k}(\mathbf{x})=0$ for $\mathbf{x} \in\left\{\mathbf{x} \mid S_{1} \mathbf{x}=0, S_{2} \mathbf{x}=0, \ldots, S_{k-1} \mathbf{x}=0\right\}$.

\section{EXAMPLE}

An example is provided for the sake of clarity. Let us consider network $\mathcal{T}=\{\{1,2,3\},\{2,3,4\},\{1,4,5\},\{1,4,6\}\}$, with $\lambda=1, \epsilon=1.5$. In the first stage of the convergence study, one spring is found and $S:=S_{1}=\left[\begin{array}{llllll}0 & 2 & -2 & 0 & 0 & 0\end{array}\right]$, $\Lambda_{1}=1$. In the second stage, we have:

$$
\begin{aligned}
S_{2} & =\left[\begin{array}{cccccc}
-1 & 0 & 0 & 1 & 0 & 0 \\
-3 & 1 / 2 & 1 / 2 & 0 & 1 & 1
\end{array}\right], \\
\Lambda_{2} & =I, \quad S:=\left[\begin{array}{l}
S_{1} \\
S_{2}
\end{array}\right] .
\end{aligned}
$$

A third stage is needed, where:

$$
\begin{aligned}
S_{3} & =\left[\begin{array}{cccccc}
1 / 14 & -5 / 14 & -5 / 14 & 1 / 14 & 2 / 7 & 2 / 7 \\
0 & 0 & 0 & 1 & -1 &
\end{array}\right], \\
\Lambda_{3} & =\left[\begin{array}{cc}
1 / 3 & 0 \\
0 & 1
\end{array}\right], S:=\left[\begin{array}{c}
S_{1} \\
S_{2} \\
S_{3}
\end{array}\right] .
\end{aligned}
$$

Since $\operatorname{ker}(S)=\operatorname{span}(\mathbf{1})$, a global second order consensus is achieved by the network under examination.

Furthermore, the convergence towards consensus is characterized by a certain degree of robustness as a result of the joint-agent interactions. In this respect, a simulation of the dynamics of this network is reported in Fig. 2, in order to highlight the potential of our study. We consider the following initial conditions: $\mathbf{x}_{0}=[2,-8,10,2,-4,4]$ and $\dot{\mathbf{x}}_{0}=[4,-6,2,-8,6,10]$. After the system has achieved a second order consensus equilibrium, agents 2 and 5 are 
simultaneously subjected to impulsive force disturbances. However, in the context of triplet $\{1,2,3\}$, the misleading action exerted by 2 is not sufficient to update the states of 1 and 3 . Similarly, in $\{2,3,4\}$ agents 3 and 4 are not sensitive to the influence of 2 , and in $\{1,4,5\}$ agents 1 and 4 are not to the one of 5 . Therefore, the second order consensus

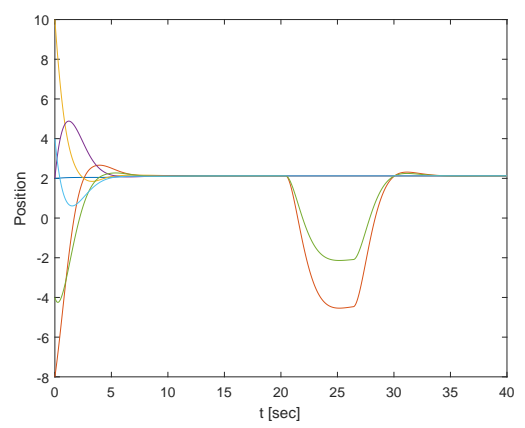

(a) Position

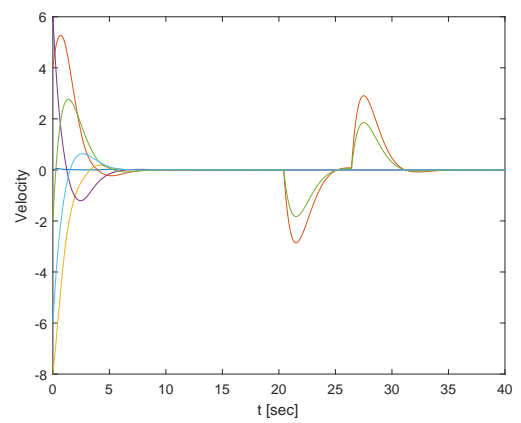

(b) Velocity

Fig. 2: Position and velocity plots for the network under examination, when agents 2 and 5 are force-disturbed.

among non-perturbed agents is not destroyed by the force disturbances on 2 and 5 . Moreover, the position and velocity states of 2 and 5 recover their respective consensus values after the disturbances have disappeared.

\section{CONCLUSiOnS}

A second order consensus protocol allowing joint-agent interactions has been proposed. The analysis is focused on networks in which agents are grouped in interaction triplets. Within a triplet, an agent is sensitive to the influence of the other members only when they exert a simultaneous and coherent action from them. This robust interaction is modelled by a piecewise linear function, which leads to some quantities being conserved in the interaction dynamics. The concept of virtual spring is defined, and a rule is provided for its detection in a general triplet-based topology. The convergence analysis is then based on the recursive detection of virtual springs, which provides asymptotically algebraic constraints to the system's dynamics as a result of damping. Our technique provides a sufficient tool for the proof of global convergence. In those network where a sufficient number of springs emerge, the global convergence towards second order consensus is proved. On the other hand, no conclusions can be drawn according to our technique when the recursive procedure is interrupted due to the lack of springs. Further studies will be devoted to extend the proposed framework, so as to enrich the class of networks that can be analysed and to attempt a formal characterization of robustness.

\section{REFERENCES}

[1] R. Olfati-Saber and J. S. Shamma, "Consensus filters for sensor networks and distributed sensor fusion," in Proceedings of the 44th IEEE Conference on Decision and Control, Dec 2005, pp. 6698-6703.

[2] S. Kar and J. M. F. Moura, "Distributed consensus algorithms in sensor networks with imperfect communication: Link failures and channel noise," IEEE Transactions on Signal Processing, vol. 57, no. 1, pp. 355-369, Jan 2009.

[3] J. Qin, W. Fu, H. Gao, and W. X. Zheng, "Distributed $k$-means algorithm and fuzzy $c$-means algorithm for sensor networks based on multiagent consensus theory," IEEE Transactions on Cybernetics, vol. 47, no. 3, pp. 772-783, March 2017.

[4] W. Ren, R. W. Beard, and E. M. Atkins, "A survey of consensus problems in multi-agent coordination," in Proceedings of the 2005, American Control Conference, 2005., June 2005, pp. 1859-1864 vol. 3.

[5] - "Information consensus in multivehicle cooperative control," IEEE Control Systems Magazine, vol. 27, no. 2, pp. 71-82, April 2007.

[6] Y. Kuriki and T. Namerikawa, "Consensus-based cooperative formation control with collision avoidance for a multi-uav system," in 2014 American Control Conference, June 2014, pp. 2077-2082.

[7] J. A. Fax and R. M. Murray, "Information flow and cooperative control of vehicle formations," IEEE Transactions on Automatic Control, vol. 49, no. 9, pp. 1465-1476, Sep. 2004.

[8] X. Dong, B. Yu, Z. Shi, and Y. Zhong, "Time-varying formation control for unmanned aerial vehicles: Theories and applications," IEEE Transactions on Control Systems Technology, vol. 23, no. 1, pp. 340348, Jan 2015.

[9] Z. Peng, G. Wen, A. Rahmani, and Y. Yu, "Distributed consensus-based formation control for multiple nonholonomic mobile robots with a specified reference trajectory," International Journal of Systems Science, vol. 46, no. 8, pp. 1447-1457, 2015. [Online]. Available: https://doi.org/10.1080/00207721.2013.822609

[10] R. Olfati-Saber and R. M. Murray, "Consensus problems in networks of agents with switching topology and time-delays," IEEE Transactions on Automatic Control, vol. 49, no. 9, pp. 1520-1533, Sep. 2004.

[11] L. Moreau, "Stability of multiagent systems with time-dependent communication links," IEEE Transactions on Automatic Control, vol. 50, no. 2, pp. 169-182, Feb 2005.

[12] Z. Lin, B. Francis, and M. Maggiore, "State agreement for continuous-time coupled nonlinear systems," SIAM Journal on Control and Optimization, vol. 46, no. 1, pp. 288-307, 2007. [Online]. Available: https://doi.org/10.1137/050626405

[13] S. Manfredi and D. Angeli, "A criterion for exponential consensus of time-varying non-monotone nonlinear networks," IEEE Transactions on Automatic Control, vol. 62, no. 5, pp. 2483-2489, May 2017.

[14] W. Ren and E. Atkins, "Distributed multi-vehicle coordinated control via local information exchange," International Journal of Robust and Nonlinear Control, vol. 17, no. 10-11, pp. 1002-1033. [Online]. Available: https://onlinelibrary.wiley.com/doi/abs/10.1002/rnc.1147

[15] W. Ren, "On consensus algorithms for double-integrator dynamics," IEEE Transactions on Automatic Control, vol. 53, no. 6, pp. 1503-1509, July 2008.

[16] W. Yu, G. Chen, M. Cao, and J. Kurths, "Second-order consensus for multiagent systems with directed topologies and nonlinear dynamics," IEEE Transactions on Systems, Man, and Cybernetics, Part B (Cybernetics), vol. 40, no. 3, pp. 881-891, June 2010.

[17] S. Manfredi and D. Angeli, "Necessary and sufficient conditions for consensus in nonlinear monotone networks with unilateral interactions," Automatica, vol. 77, pp. 51-60, 032017.

[18] D. Angeli and S. Manfredi, "On consensus protocols allowing joint-agent interaction," IEEE Conference on Decision and Control, submitted.

[19] D. Angeli and S. Manfredi, "On Adversary Robust Consensus protocols through joint-agent interactions," arXiv e-prints, p. arXiv:1901.02725, Jan 2019. 